\title{
The saponin monomer of dwarf lilyturf tuber, DT-13, inhibits angiogenesis under hypoxia and normoxia via multi-targeting activity
}

\author{
RENPING ZHAO ${ }^{1 *}$, LI SUN $^{2 *}$, SENSEN LIN ${ }^{1}$, XIANSHU BAI ${ }^{4}$, BOYANG YU ${ }^{5}$, \\ SHENGTAO YUAN $^{1}$ and LUYONG ZHANG ${ }^{2,3}$
}

\begin{abstract}
${ }^{1}$ Jiangsu Center for Pharmacodynamics Research and Evaluation, ${ }^{2}$ Jiangsu Center for Drug Screening, China Pharmaceutical University; ${ }^{3}$ Key Laboratory of Drug Quality Control and Pharmacovigilance (China Pharmaceutical University), Ministry of Education, Nanjing, Jiangsu 210009, P.R. China; ${ }^{4}$ Department of Molecular Biology, University of Saarland, D-66421 Homburg, Germany; ${ }^{5}$ Department of Complex Prescription of Traditional Chinese Medicine, School of Chinese Material Medicine, China Pharmaceutical University, Nanjing, Jiangsu 210009, P.R. China
\end{abstract}

Received October 31, 2012; Accepted November 26, 2012

DOI: $10.3892 /$ or.2013.2272

\begin{abstract}
The saponin monomer of dwarf lilyturf tuber, DT-13, exhibits anticancer activity by reducing human breast cancer cell adhesion and migration under hypoxia. To further investigate the anticancer activity of DT-13, we investigated whether DT-13 exhibits anti-angiogenic activity. DT-13 showed no effect on human umbilical vein endothelial cell proliferation but inhibited tube formation and migration under normoxia and hypoxia. Moreover, DT-13 significantly reduced density of vessels in vivo observed from a chicken chorioallantoic membrane model. Western blotting results showed that DT-13 suppressed the increased level of hypoxia-inducible factor $1 \alpha$, p-extracellular signal-regulated kinase 1/2 and p-Akt induced by hypoxia. Enzyme-linked immunosorbent assay revealed that vascular endothelial growth factor excretion was suppressed by DT-13. DT-13 inhibited migration and tube formation induced by vascular endothelial growth factor under normoxia and hypoxia. In addition, DT-13 reduced the level of p-vascular endothelial growth factor receptor 2 and p-Akt induced by vascular endothelial growth factor. Our data suggest that DT-13 inhibits angiogenesis under normoxia and hypoxia and also inhibits angiogenesis induced by vascular endothelial growth factor via targeting at multi elements.
\end{abstract}

Correspondence to: Professor Shengtao Yuan, Jiangsu Center for Pharmacodynamics Research and Evaluation, China Pharmaceutical University, 24 Tongjiaxiang, Nanjing, Jiangsu 210009, P.R. China

E-mail: yuanst2@yahoo.com.cn

Professor Luyong Zhang, Jiangsu Center for Drug Screening, China Pharmaceutical University, 24 Tongjiaxiang, Nanjing, Jiangsu 210009, P.R. China

E-mail: drugscreen@163.com

${ }^{*}$ Contributed equally

Key words: DT-13, dwarf lilyturf tuber, hypoxia, normoxia, angiogenesis, vascular endothelial growth factor, human umbilical vein endothelial cells

\section{Introduction}

Aside from tumor cell proliferation, expansion and metastasis, angiogenesis is one of the essential steps in tumor progression. When the diameter is over $0.5 \mathrm{~mm}$, the tumor stimulates blood vessel growth to cover the need for nutrition and oxygen, known as the process of angiogenesis (1). The correlation between angiogenesis and tumor metastasis has been described in two points indicating that angiogenesis plays a key role in tumor metastasis: first, the increased vascularity of the primary tumor always leads to increased number of metastatic colonies; second, increased vascular density is indicative of increased metastasis colony size (2). Thus, antiangiogenesis is one of the crucial targets of tumor therapy and anti-angiogenic therapy has been used to inhibit cancer growth and metastasis in the clinic (3). For instance, bevacizumab, the first anti-angiogenic drug approved by the FDA, is a monoclonal antibody against vascular endothelial growth factor (VEGF)-A, inhibiting tumor growth and metastasis $(4,5)$ via anti-angiogenesis $(6)$. Various factors induce angiogenesis, including hypoxia. In tumor, due to the accelerated proliferation of tumor resulting in an abnormal and chaotic blood supply, blood vessels cannot provide oxygen adequately or consistently to the whole tumor. Moreover, the temporal changes in blood flow cause endothelium to suffer hypoxia in certain regions (7).

Dwarf lilyturf tuber, a traditional Chinese medicine, is generally used for cardiovascular disease treatment (8). It has been proved that the saponin monomer of dwarf lilyturf tuber, DT-13, exerts anticancer activity $(9,10)$. It has also been reported that some saponin, such as ginseng saponin, could inhibit angiogenesis $(11,12)$. Previous studies on cancer cell lines indicated that DT-13 inhibits MDA-MB-435 cell migration and adhesion. DT-13 downregulates early growth response gene-1 expression resulting in the reduced excretion of tissue factor under hypoxia (13). In addition, DT-13 decreases the excretion and expression of matrix metallopeptidase 2/9 (MMP-2/9) and reduces the phosphorylation of p38 
(14). However, the effect of DT-13 on angiogenesis remains unclear. In the present study, we investigated if DT-13 impacts angiogenesis under normoxia or hypoxia and we examined the potential mechanisms.

\section{Materials and methods}

Cell culture. Human umbilical vein endothelial cells (HUVECs) were isolated as previously described (15). HUVECs were cultured in complete medium consisting of M199 medium (Gibco), 20\% FBS, $50 \mathrm{ng} / \mathrm{ml}$ of endothelial cell growth supplement (ECGS, Sigma) and $100 \mathrm{ng} / \mathrm{ml}$ heparin. Endothelial cells from third to sixth passages were used for experiments. The cells were incubated in a humidified atmosphere of $95 \%$ air, $5 \% \mathrm{CO}_{2}$ at $37^{\circ} \mathrm{C}$. For hypoxic treatment, we placed $70-80 \%$ confluent cells in a tissue culture incubator in an atmosphere of $1 \% \mathrm{O}_{2}$ and $5 \% \mathrm{CO}_{2}$ at $37^{\circ} \mathrm{C}(16)$.

HUVEC proliferation assay. HUVECs were harvested, washed with phosphate buffer solution (PBS), re-suspended in complete medium and plated in 96-well plates at the density of $5 \times 10^{3}$ cells/well, followed by a 12 -h incubation. Cells were then treated with $10^{-8}, 10^{-7}$ and $10^{-6} \mathrm{M}$ DT-13 (provided by Professor Bo-Yang Yu) (Fig. 1) for 24 and $48 \mathrm{~h}$ under normoxia. The cell viability was evaluated by the Cell Counting kit (CCK-8, Dojindo Laboratories), or the medium was changed to ECGSfree medium (M199 medium with 1\% FBS without ECGS). HUVECs were pretreated with $10^{-8}, 10^{-7}$ and $10^{-6} \mathrm{M}$ DT-13 for $1 \mathrm{~h}$, and were then treated with $10 \mathrm{ng} / \mathrm{ml}$ VEGF for $48 \mathrm{~h}$. Following treatment, the cell viability was evaluated by the Cell Counting kit.

Cell migration assay. The cell migration assay was performed by using a Transwell chamber migration system with polycarbonate membranes ( $8.0 \mu \mathrm{M}$ pore size), as previously described (17). Briefly, HUVECs were harvested, re-suspended in serum-free culture medium and $\left(2 \times 10^{4}\right.$ cells/well) seeded with various concentrations of DT-13 into the upper chamber. Complete culture medium was added into the lower chamber. The cell migration system was incubated at $37^{\circ} \mathrm{C}$ for $8 \mathrm{~h}$, or, HUVECs were harvested, re-suspended with ECGS-free medium and plated $\left(2 \times 10^{4}\right.$ cell/well $)$ in the presence of various concentrations of DT-13 into the upper chamber. VEGF $(10 \mathrm{ng} / \mathrm{ml})$ in ECGS-free medium was added into the lower chamber. The cell migration system was incubated at $37^{\circ} \mathrm{C}$ for $4 \mathrm{~h}$. Following treatment, cells were stained and counted in three randomly selected fields.

Tube formation assay. Tube formation assay was performed as previously described (18). Briefly, 96-well plates were coated with Matrigel $\left(50 \mu 1 /\right.$ well), and incubated at $37^{\circ} \mathrm{C}$ for $1 \mathrm{~h}$ to solidify Matrigel. HUVECs $\left(1.5 \times 10^{4}\right.$ cells/well $)$ were incubated with various concentrations of DT- 13 at $37^{\circ} \mathrm{C}$. After $6 \mathrm{~h}$, tubular structures were quantified by manual counting of the number of tubes in three randomly selected fields. When HUVECs were treated with $10 \mathrm{ng} / \mathrm{ml}$ of VEGF, the growth factors reduced Matrigel and ECGS-free medium was preferred, and HUVECs were incubated under normoxia. If not, basement Matrigel and complete medium was preferred, and HUVECs were incubated under hypoxia and normoxia.

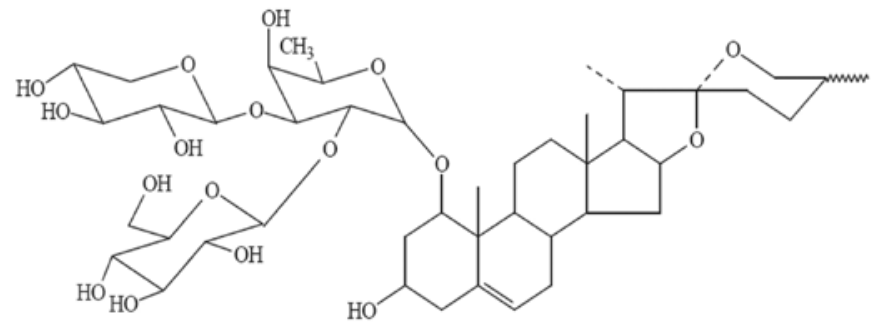

Figure 1. Chemical structure of the saponin monomer of dwarf lilyturf tuber (DT-13).

Chicken embryo chorioallantoic membrane (CAM) assay. A small window was created on the broad side of the 5-dayold egg. The filter paper with different doses of DT-13 (final concentration $0.001,0.01$ and $0.1 \mathrm{nmol} / \mathrm{egg}$ ) was dropped on the window. The eggs were incubated at $37^{\circ} \mathrm{C}$ for another 2 days. We then observed the density and length of vessels toward the CAM face and then the CAMs were photographed. Eight eggs were used per group, and the newly formed vessels were counted.

Western blot analysis. Cellular protein extraction and western blot analysis were performed as previously described (16). Briefly, $40 \mu \mathrm{g}$ total protein was fractionated using $10 \%$ sodium dodecyl sulfate polyacrylamide gel electrophoresis (SDS-PAGE) and transferred onto nitrocellulose membranes under semi-dry conditions. Membranes were probed with rabbit anti-human p-extracellular signal-regulated kinase (ERK) 1/2 (CST), ERK1/2 (CST), p-AKT (CST), AKT (CST), HIF-1 $\alpha$ (Santa Cruz), and murine anti-human $\beta$-actin (Sigma). Horseradish peroxidase (HRP) linked anti-mouse immunoglobulin G (Sigma) and anti-rabbit immunoglobulin G (CST) were used as the secondary antibodies. Protein bands were visualized by enhanced chemiluminescence reagents (Amersham Pharmacia Biotech).

Enzyme-linked immunosorbent assay (ELISA). HUVECs were plated into $60-\mathrm{mm}$ dishes with completed medium overnight. HUVECs were changed medium to serum and growth factor supplement-free medium. After $2 \mathrm{~h}$, cells were treated with various concentrations of DT-13 for $12 \mathrm{~h}$ under hypoxia or normoxia. After treatment, the medium was harvested to detect the concentration of VEGF by ELISA. The level of VEGF in HUVEC medium was measured with a VEGF ELISA kit (4A Biotech) according to the manufacturer's protocol.

Statistical analysis. The data represent at least three independent experiments and are expressed as the means \pm SEM. For statistical analysis, the Student's t-test was used as appropriate.

\section{Results}

Effect of DT-13 on HUVEC proliferation. To identify the cytotoxicity of DT-13, we treated HUVECs with $0.01,0.1$ and $1 \mu \mathrm{M}$ DT-13 for 24 and $48 \mathrm{~h}$. DT-13 at $1 \mu \mathrm{M}$ inhibited cell proliferation to 71 and $86 \%$ of control at 24 and $48 \mathrm{~h}$ of treatment, respectively (Fig. 2A and B). However, $0.01 \mu \mathrm{M}$ of DT-13 slightly improved HUVEC proliferation after $48 \mathrm{~h}$ of 
A
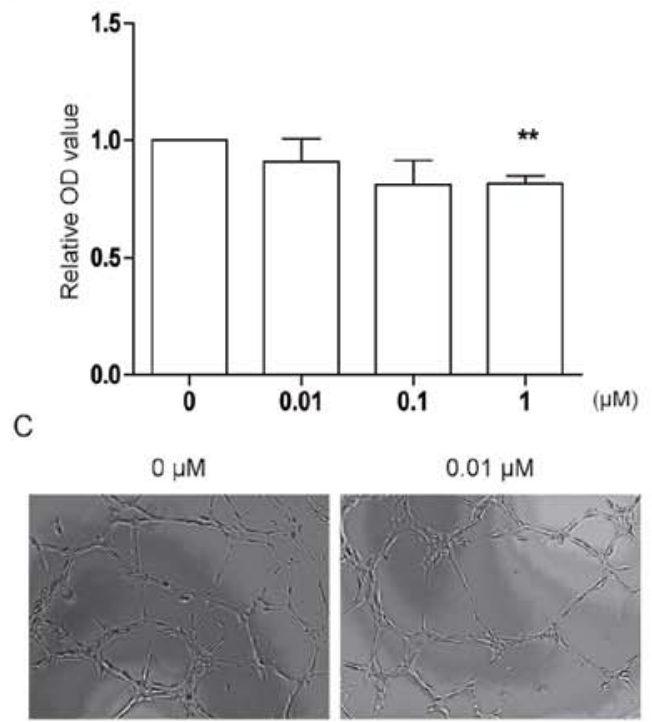

$0.1 \mu \mathrm{M}$

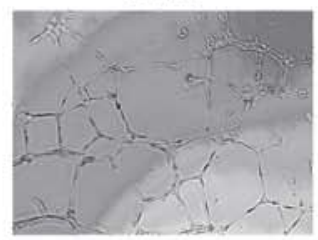

E

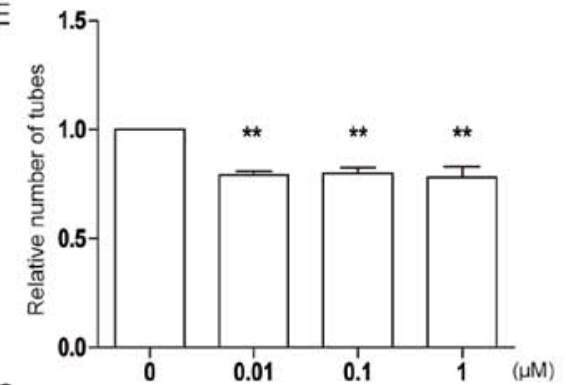

B

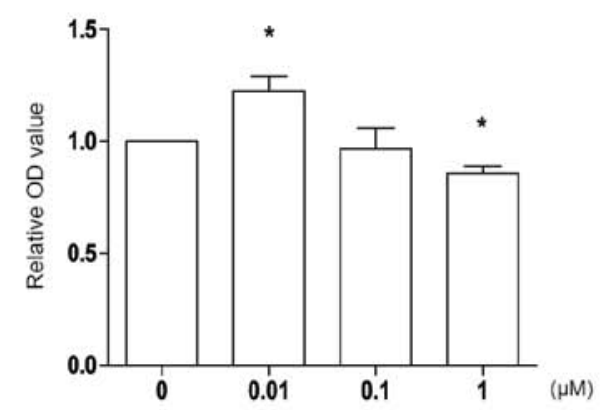

D
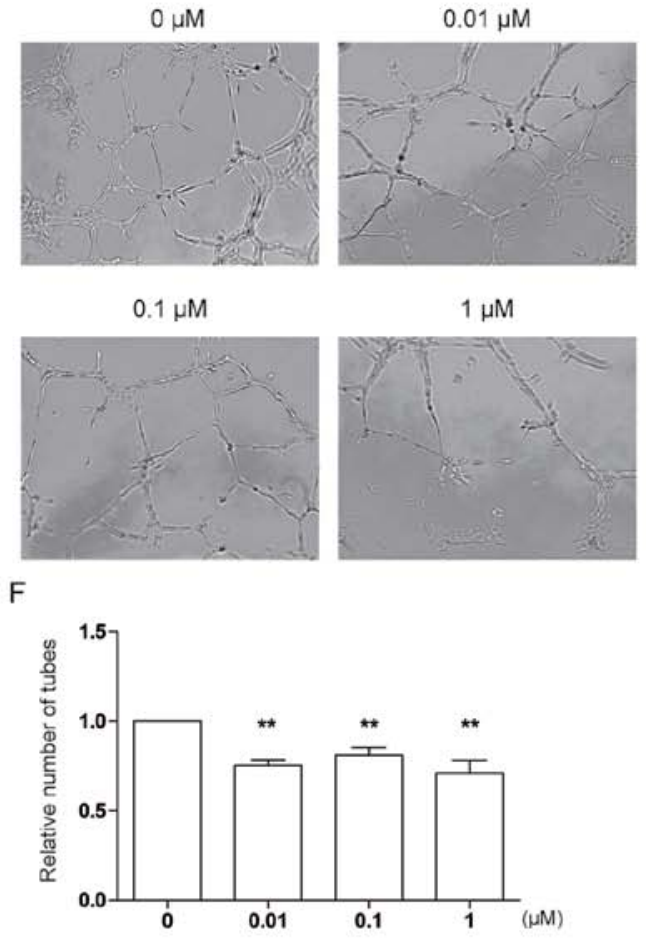

Normoxia

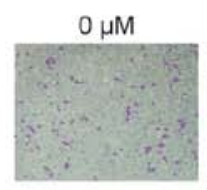

Hypoxia

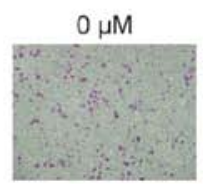

$0.01 \mu \mathrm{M}$

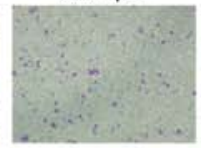

$0.01 \mu \mathrm{M}$

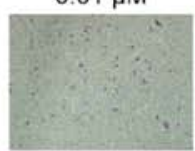

$0.1 \mu \mathrm{M}$

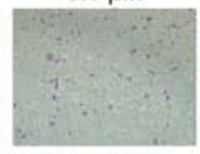

$0.1 \mu \mathrm{M}$

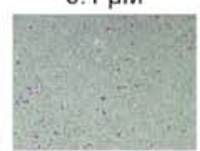

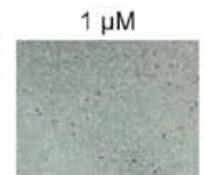

$1 \mu \mathrm{M}$
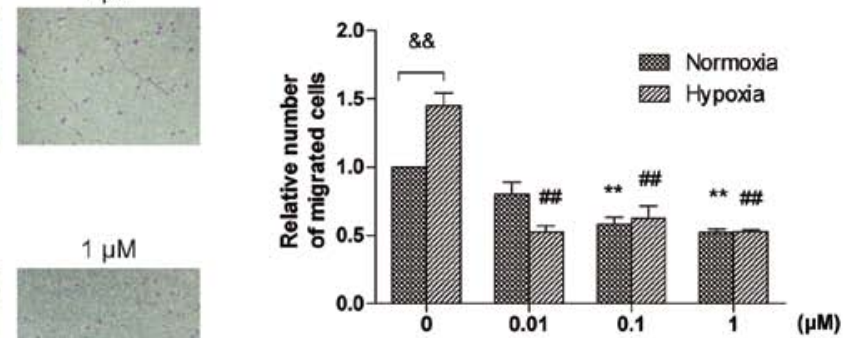

Figure 2. DT-13 inhibits angiogenesis under normoxia and hypoxia in vitro. (A) The effect of DT-13 on HUVEC proliferation for 24 h under normoxia. HUVECs were treated with DT-13 $(0.01,0.1$ and $1 \mu \mathrm{M})$ or PBS for $24 \mathrm{~h}$ under normoxia. Following treatment, the number of cells was counted by CCK-8. ${ }^{* *} \mathrm{p}<0.01$, statistical significance relative to vehicle control. (B) The effect of DT-13 on HUVEC proliferation for $48 \mathrm{~h}$ under normoxia. HUVECs were treated with DT-13 $(0.01,0.1$ and $1 \mu \mathrm{M})$ or PBS for $48 \mathrm{~h}$ under normoxia. Following treatment, the number of cells was counted by CCK-8. ${ }^{*}$ p $<0.05$, statistical significance relative to vehicle control. (C and E) The effect of DT-13 on tube formation under normoxia. HUVECs were plated into 96-well plates pre-coated by Matrigel and treated with DT-13 $(0.01,0.1$ and $1 \mu \mathrm{M})$ or PBS. HUVECS were incubated under normoxia for $6 \mathrm{~h}$. After treatment, the tubes were captured and counted. ${ }^{* *} \mathrm{p}<0.01$, statistical significance relative to vehicle control. (D and F) The effect of DT-13 on tube formation under hypoxia and normoxia. HUVECs were plated into 96-well plates pre-coated by Matrigel and treated with DT-13 $(0.01,0.1$ and $1 \mu \mathrm{M})$ or PBS. HUVECS were incubated under hypoxia for 6 h. After treatment, the tubes were captured and counted. ${ }^{* *} \mathrm{p}<0.01$, statistical significance relative to vehicle control. (G) The effect of DT-13 on HUVEC migration under normoxia and hypoxia. HUVECs in serum-free medium with DT-13 $(0.01,0.1$ and $1 \mu \mathrm{M})$ or PBS were plated into the inner chamber of Transwell and complete medium was added into the outer chamber. The Transwell system was incubated under normoxia or hypoxia for $8 \mathrm{~h}$. After treatment, the cells on the outer side membrane were fixed and stained by crystal violet. These cells were captured and counted. ${ }^{* *}$ p $<0.01$, statistical significance relative to vehicle control under normoxia. ${ }^{\# \#} \mathrm{p}<0.01$, statistical significance relative to vehicle control under hypoxia. \&\&p $<0.01$, statistical significance relative to vehicle control under hypoxia. 
treatment. These results indicate that DT-13 inhibits HUVEC proliferation at high concentrations but promotes proliferation at low concentrations.

DT-13 inhibits tube formation and HUVEC migration under hypoxia and normoxia. To further investigate the effect of DT-13 on angiogenesis, we performed tube formation and migration assays under normoxic and hypoxic conditions, since DT-13 displayed marked anticancer activity under hypoxia (13). In the tube formation study, HUVECs were treated with $0.01,0.1$ and $1 \mu \mathrm{M}$ DT-13 and incubated under normoxia or hypoxia for $6 \mathrm{~h}$. Under normoxia, different concentrations of DT-13 showed similar inhibitory effects on tube formation by $20 \%$ compared with control $(21,20$ and $21 \%$ by $0.01,0.1$ and $1 \mu \mathrm{M}$ of DT-13 respectively) (Fig. 2C and E). Under hypoxia, the inhibitory activity was 25,19 and $29 \%$ compared with control, respectively (Fig. 2D and F).

In the migration assay, we planted HUVECs into the upper chamber of Transwell with serum-free medium in the presence or absence of DT-13, and added complete medium into the lower chamber. Under normoxia, DT-13 reduced the number of migrated cells with the inhibitory rate of 20, 40 and $48 \%$ at the concentration of $0.01,0.1$ and $1 \mu \mathrm{M}$, respectively (Fig. 2G). Under hypoxia, DT-13 performed stronger activity on inhibiting migration; different concentrations of DT-13 showed similar inhibitory effects on migration by $40 \%$ compared with control (36, 43 and $36 \%$ by $0.01,0.1$ and $1 \mu \mathrm{M}$ of DT-13 respectively) (Fig. 2G). These results indicate that DT-13 inhibits tube formation and migration under normoxia and this effect is stronger under hypoxia.

DT-13 inhibits angiogenesis in vivo. To confirm the antiangiogenesis activity of DT-13 in vivo, we used a CAM model. Five-day-old eggs were treated with PBS or DT-13 $(0.1,0.01$ and $0.001 \mathrm{nmol} / \mathrm{egg}$ ) for $48 \mathrm{~h}$ under normoxia. The normal branching pattern of blood vessels formed after 2 days of incubation. As shown in Fig. 3, DT-13 significantly reduced the relative length of vessels. Different concentrations of DT-13 showed similar inhibitory rates approximately $35 \%$ compared with PBS. These data suggest that DT-13 inhibits angiogenesis in vivo with a large spectrum of dose.

DT-13 downregulates the level of HIF-1 $\alpha, p-E R K$, and $p$-Akt under hypoxia. Hypoxia-inducible factor $1 \alpha$ (HIF-1 $\alpha$ ) accumulates under hypoxia and is a crucial factor for angiogenesis (19). Thus, we investigated if DT-13 affects the level of HIF-1 $\alpha$. Hypoxia strongly upregulated HIF-1 $\alpha$ expression compared with normoxia (Fig. 4A). However, DT-13 decreased the amount of HIF-1 $\alpha$ both under normoxia and hypoxia. Under hypoxia, DT-13 at the concentrations of 0.01 and $1 \mu \mathrm{M}$ significantly downregulated increased HIF-1 $\alpha$ induced by hypoxia. Moreover, DT-13 significantly downregulated ERK1/2 and Akt phosphorylation in a dose-dependent manner under hypoxia (Fig. 4A). Under normoxia, DT-13 only slightly reduced the level of p-Akt at $1 \mu \mathrm{M}$ (Fig. 4A). These data suggest that DT-13 exerts anti-angiogenic effects under hypoxia by inhibiting ERK1/2 and Akt phosphorylation.

DT-13 inhibits VEGF excretion under hypoxia. We also detected whether DT-13 impacts VEGF secretion under normoxia and
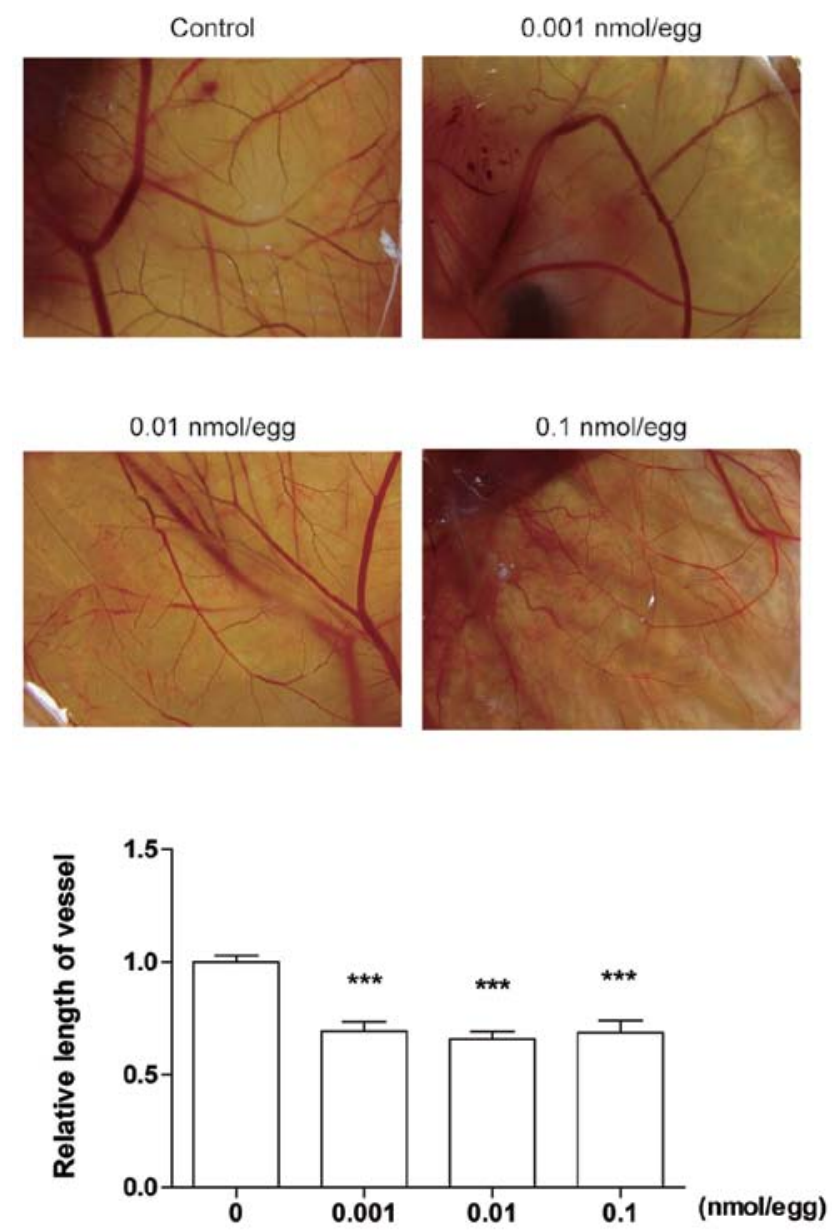

Figure 3. The effect of DT-13 on new blood vessel formation in CAM models Filter paper with DT-13 (0.001, 0.01 and $0.1 \mathrm{nmol} / \mathrm{egg})$ or PBS was plated on chicken embryo chorioallantoic membranes of 5-day eggs, then incubated at $37^{\circ} \mathrm{C}$ for 2 days. ${ }^{* * *} \mathrm{p}<0.001$, statistical significance relative to vehicle control.

hypoxia with the ELISA assay. The medium of HUVECs was changed to serum-free medium and incubated with or without DT-13 under normoxia or hypoxia for $12 \mathrm{~h}$. The conditioned medium was collected and examined. As shown in Fig. 4B, under normoxia DT-13 decreased VEGF secretion in a dosedependent manner with the inhibition rate of 11,15 and $40 \%$ by $0.01,0.1$ and $1 \mu \mathrm{M}$ of DT-13. Under hypoxia, DT-13 always showed similar inhibitory rates by $40 \%$ compared with control (37, 42 and $38 \%$ by $0.01,0.1$ and $1 \mu \mathrm{M}$ of DT- 13 respectively) (Fig. 4B).

DT-13 inhibits angiogenesis induced by VEGF. As shown above, DT-13 exhibited anti-angiogenic ability and inhibited VEGF excretion under normoxia and hypoxia, thus we investigated whether DT-13 inhibits angiogenesis induced by VEGF. Initially, DT-13 did not inhibit cell proliferation at the presence of VEGF within $48 \mathrm{~h}$ (Fig. 5A). However, DT-13 exhibited anti-angiogenic effects observed from cell migration and tube formation assays. DT-13 reduced the number of migrated cells induced by VEGF with the inhibitory rate of $61.5,40.1$ and $38.9 \%$ at the concentration of $0.01,0.1$ and $1 \mu \mathrm{M}$ respectively (Fig. 5B), and decreased tube formation to 63.4, 49.3 and 35\% of control at the concentration of $0.01,0.1$ and $1 \mu \mathrm{M}$ (Fig. 5C). 
A
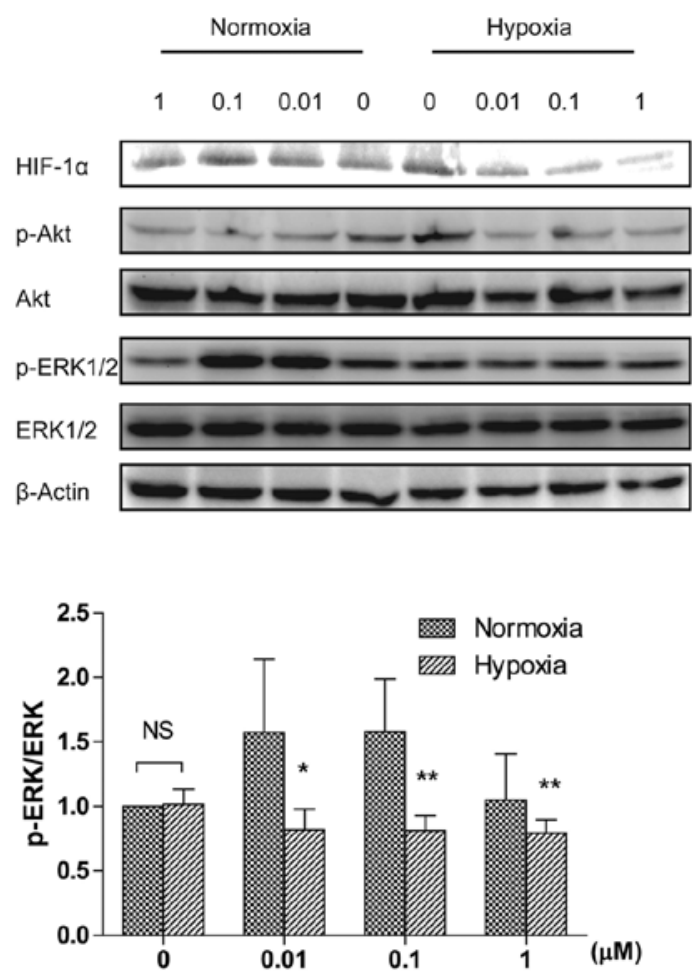

B

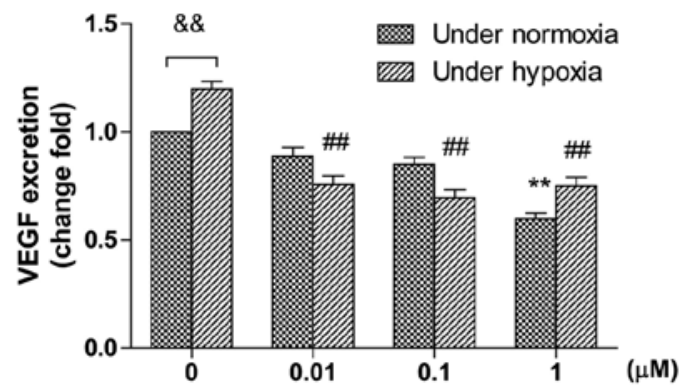

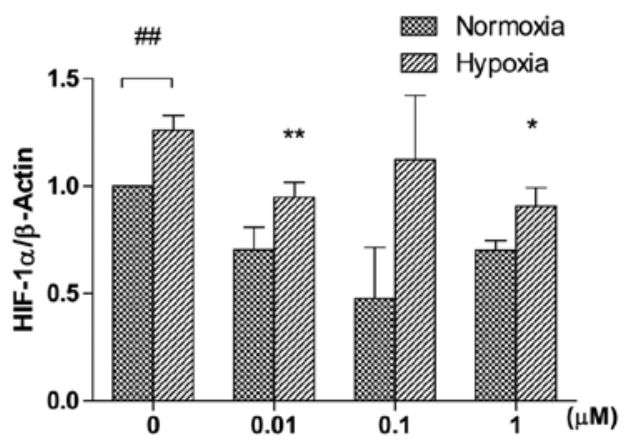

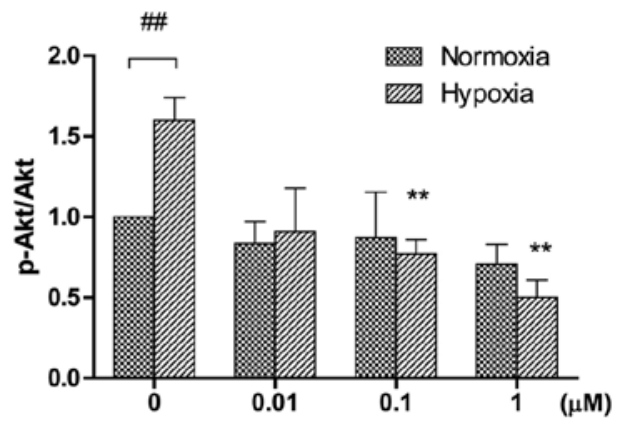

Figure 4. (A) The effect of DT-13 on ERK1/2 and Akt activation and the level of HIF-1 $\alpha$ under normoxia and hypoxia. Western blot analysis demonstrated that DT-13 inhibits phosphorylation of ERK1/2, Akt and HIF-1 $\alpha$. Total cell lysate was mixed with SDS loading buffer and separated by SDS-PAGE, blotted onto nitrocellulose membranes. Membranes were immunoblotted with the respective primary antibodies: p-ERK1/2, ERK1/2, p-Akt, Akt, HIF-1 $\alpha$ and $\beta$-actin. ${ }^{*} \mathrm{p}<0.05 ;{ }^{* *} \mathrm{p}<0.01$, statistical significance relative to vehicle control under hypoxia. ${ }^{\# \#} \mathrm{p}<0.01$, statistical significance relative to vehicle control under normoxia. (B) The effect of DT-13 on VEGF secretion under normoxia and hypoxia. HUVECs were plate into 60-mm dishes with completed medium overnight. HUVECs were changed medium to serum and growth factor supplement-free medium. After $2 \mathrm{~h}$, cells were treated with various concentrations of DT-13 for $12 \mathrm{~h}$ under hypoxia or normoxia. After treatment, the medium was harvested to detect the concentration of VEGF by ELISA. ${ }^{* *}$ p $<0.01$, statistical significance relative to vehicle control under normoxia. ${ }^{\# \#} \mathrm{p}<0.01$, statistical significance relative to vehicle control under hypoxia. \&\& $\mathrm{p}<0.01$, statistical significance relative to vehicle control under hypoxia.

The results indicate that DT-13 attenuates the effect of VEGF on angiogenesis by inhibiting HUVEC migration and tube formation, but does not affect HUVEC proliferation induced by VEGF.

DT-13 exhibits anti-angiogenic activity by inhibiting the VEGF pathway. As shown above, angiogenesis induced by VEGF is weakened by DT-13. Therefore, we evaluated if DT-13 affects the downstream of VEGF pathway. Firstly, we detected the phosphorylation of VEGFR2, the receptor of VEGF. We pretreated HUVECs with or without DT-13 for $1 \mathrm{~h}$ and then treated with $10 \mathrm{ng} / \mathrm{ml} \mathrm{VEGF}$ for $15 \mathrm{~min}$. VEGF significantly promoted the phosphorylation of VEGFR2 compared with PBS, while DT-13 decreased the level of p-VEGFR2 induced by VEGF, with a significant difference at the concentration of $1 \mu \mathrm{M}$ (Fig. 6). Furthermore, we examined the phosphorylation of Akt and ERK1/2 after VEGF and DT-13 treatment. VEGF increased the level of p-Akt, which was markedly attenuated by $1 \mu \mathrm{M}$ DT-13 (Fig. 6). However, increased p-ERK1/2 was slightly decreased by DT-13 without statistical difference (Fig. 6). These results suggest that under hypoxia, DT-13 inhibits angiogenesis by inhibiting the VEGF pathway.

\section{Discussion}

In this study, we demonstrated that DT-13 exhibits antiangiogenic activity under hypoxia and normoxia, and inhibits angiogenesis induced by VEGF under normoxia in vitro. DT-13 


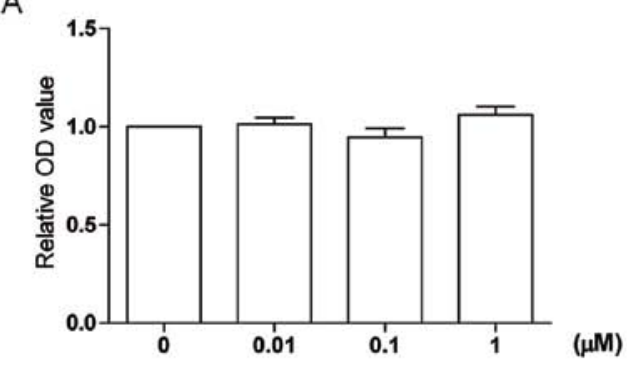

B

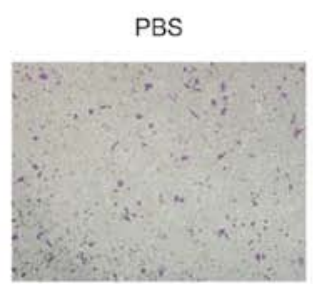

VEGF+0.01 $\mu \mathrm{M}$

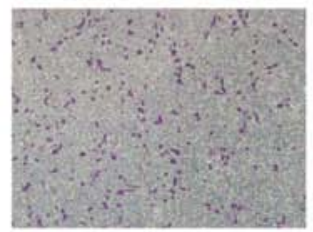

VEGF+1 $\mu \mathrm{M}$
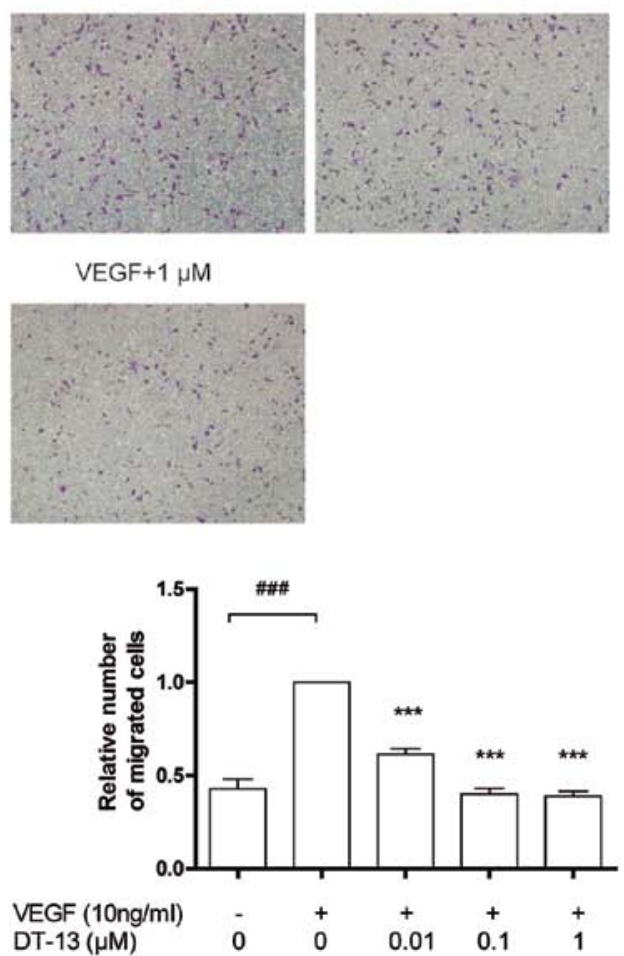

C
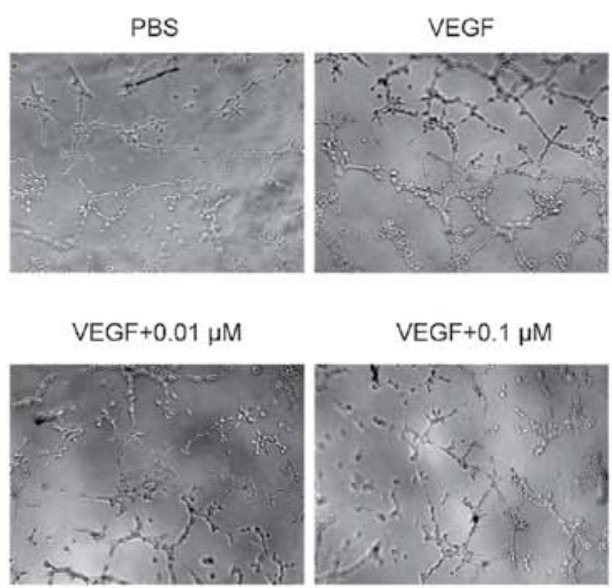

VEGF+1 $\mu \mathrm{M}$
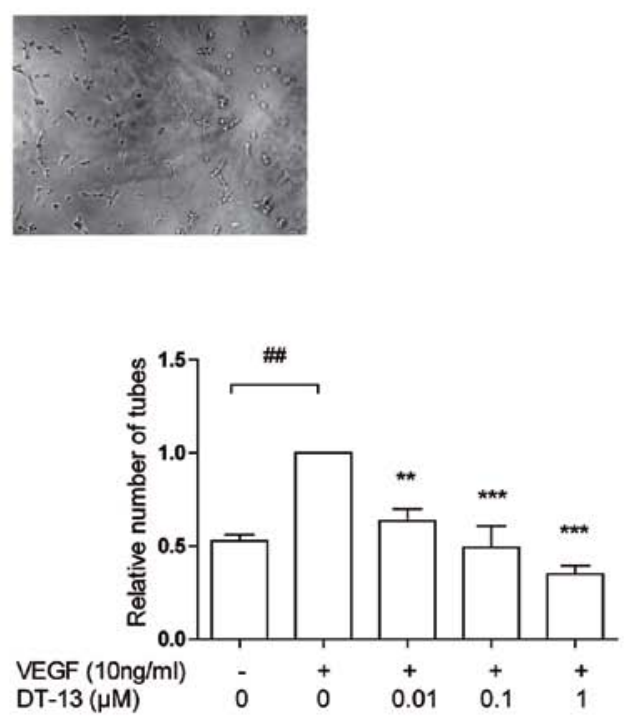

Figure 5. DT-13 inhibits angiogenesis stimulated by VEGF in vitro. (A) The effect of DT-13 on HUVEC proliferation stimulated by VEGF for 48 h under normoxia. HUVECs were pre-treated with DT-13 $(0.01,0.1$ and $1 \mu \mathrm{M})$ or PBS for $1 \mathrm{~h}$, then treated with $10 \mathrm{ng} / \mathrm{ml} \mathrm{VEGF}$ for $48 \mathrm{~h}$. After treatment, the number of cells was counted by CCK-8. (B) The effect of DT-13 on HUVEC migration stimulated by VEGF. HUVECs in $1 \%$ FBS and ECGS-free medium with DT-13 $(0.01,0.1$ and $1 \mu \mathrm{M})$ or PBS were plated into the inner chamber of Transwell and $1 \%$ FBS and ECGS-free medium plus 10 ng/ml VEGF was added into the outer chamber. The Transwell system was incubated for $4 \mathrm{~h}$. After treatment, the cells on the outer side membrane were fixed and stained by crystal violet. These cells were captured and counted. ${ }^{* * * *}$ p $<0.001$, statistical significance relative to the VEGF plus group. ${ }^{\# \# \#}$ p $<0.001$, statistical significance relative to the VEGF-free group. (C) The effect of DT-13 on tube formation stimulated by VEGF. HUVECs in $1 \%$ FBS and ECGS-free medium were plated into 96-well plates pre-coated by growth factor reduced Matrigel and treated with VEGF $(10 \mathrm{ng} / \mathrm{ml})$, DT-13 $(0.01,0.1$ and $1 \mu \mathrm{M})$ or PBS. HUVECS were incubated for $6 \mathrm{~h}$. After treatment, the tubes were captured and counted. ${ }^{* *} \mathrm{p}<0.01 ;{ }^{* * *} \mathrm{p}<0.001$, statistical significance relative to the VEGF plus group. ${ }^{\# \#} \mathrm{p}<0.01$, statistical significance relative to the VEGF-free group.

administration resulted in a significant reduction of HUVEC proliferation, migration and tube formation under hypoxia and normoxia. Further evaluation with CAM likely results in reduced relative vessel length in DT-13-treated eggs. To address the potential mechanism of the anti-angiogenic effects of DT-13, we detected the elements involved in angiogenesis, such as HIF- $1 \alpha$,
p-Akt, and ERK1/2 which showed significantly decreased levels after DT-13 treatment under hypoxia but not under normoxia. Additionally, DT-13 reduced VEGF secretion and VEGF induced p-VEGFR2 and p-Akt. These findings indicate a role for DT-13 in the inhibition of angiogenesis under hypoxia and normoxia or even of angiogenesis induced by VEGF. 

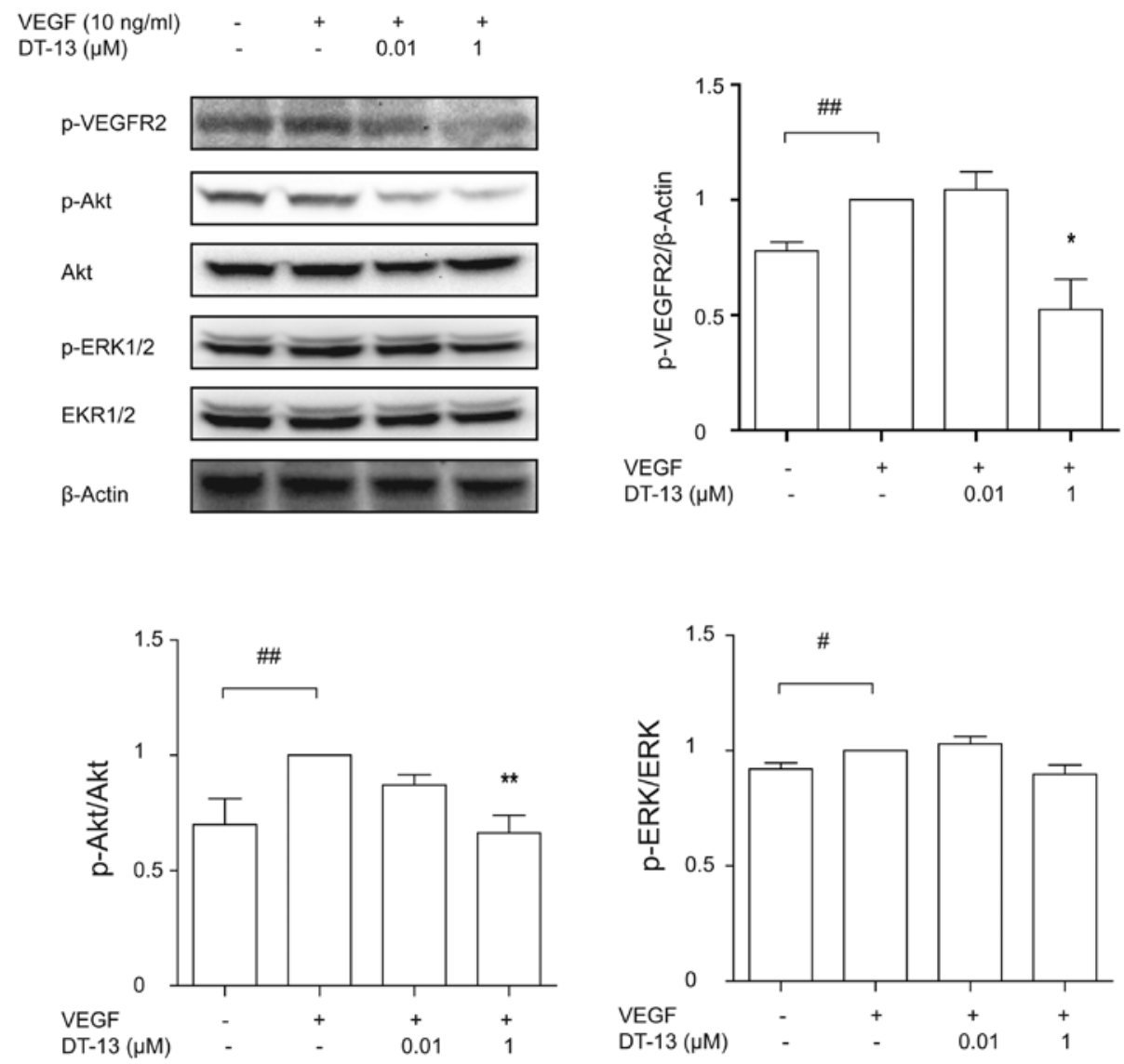

Figure 6. The effect of DT-13 on VEGF-stimulated activation of VEGFR-2 and related signaling pathways. Western blot analysis demonstrated that DT-13 inhibits phosphorylation of VEGFR2, ERK1/2 and Akt. Total cell lysate was mixed with SDS loading buffer and separated by SDS-PAGE, blotted onto nitrocellulose membranes. Membranes were immunoblotted with the respective primary antibodies: p-ERK1/2, ERK1/2, p-Akt, Akt, p-VEGFR2 and $\beta$-actin. ${ }^{*} \mathrm{p}<0.05 ;{ }^{* *} \mathrm{p}<0.01$, statistical significance relative to the VEGF plus group. ${ }^{\#} \mathrm{p}<0.05 ;{ }^{\# \#} \mathrm{p}<0.01$, statistical significance relative to the VEGF-free group.

At present, there are three types of anti-angiogenesis drugs: antibody, synthetic small molecules, and natural products (20). Although the effects of the anti-VEGF-A antibody, bevacizumab, have been confirmed in clinical anti-angiogenic therapy combined with a cytotoxic agent (21), there are still adverse effects in the cardiovascular and renal systems $(22,23)$. Multiple-target small molecules and natural products performed anti-angiogenic activity, such as sorafenib and curcumin (20). Previous studies on cancer cells suggested that DT-13 exhibits anticancer activity via inhibiting cancer cell migration and adhesion in vitro $(13,14)$. Our data showed that DT-13 displays anti-angiogenic activity by inhibiting tube formation and HUVEC migration. Endothelial cells in tumor are under two main oxygen conditions, normoxia and hypoxia. Endothelial cells are under hypoxia during the pathological state, such as atherosclerosis and cancer (19,24-26). Hypoxic conditions stimulate the expression of pro-angiogenic protein, such as VEGF and its receptor VEGFR2, in endothelial cells (27-29). VEGFR-2 plays a crucial role in angiogenesis and activation of VEGFR-2 promotes endothelial cell growth, migration and tube formation (30). In VEGFR-2-deficient mice, failed formation of blood islands and blood vessels led to embryonic lethality (31), which indicates that VEGFR-2 is essential in angiogenesis. Stimulation of VEGFR-2 phosphorylates downstreams, such as ERK1/2 (32) and Akt (33). DT-13 reduces the level of p-Akt and ERK1/2 under hypoxia and p-VEGFR-2 and p-Akt induced by
VEGF under normoxia, but displays little effect on p-ERK1/2 induced by VEGF. DT-13 displays higher activity of inhibition on p-Akt and p-ERK1/2 under hypoxia than under normoxia. Furthermore, DT-13 reduces the increased HIF-1 $\alpha$ induced by hypoxia, which may also reduce angiogenesis considering the role of HIF-1 $\alpha$ in angiogenesis (19). Thus, DT-13 could be a new multi-targeting anticancer agent by inhibiting angiogenesis, tumor growth and metastasis.

In conclusion, we provided evidence for the first time that DT-13 exhibits anti-angiogenic activity. Further animal studies are required to confirm the effect of DT-13 on angiogenesis and to explore the side-effects of DT-13.

\section{Acknowledgements}

This study was financially supported by the National Natural Science Fund Nos. 81102853 and 81071841, and 2011' Program for Excellent Scientific and Technological Innovation Team of Jiangsu Higher Education.

\section{References}

1. Bamias A and Dimopoulos MA: Angiogenesis in human cancer: implications in cancer therapy. Eur J Intern Med 14: 459-469, 2003.

2. Zetter BR: Angiogenesis and tumor metastasis. Annu Rev Med 49: 407-424, 1998 . 
3. He K, Jin K, Wang $\mathrm{H}$ and Teng L: Anti-angiogenic therapy for colorectal cancer: on the way to getting better! Hepatogastroenterology 59: 1113-1117, 2012.

4. Das $M$ and Wakelee H: Targeting VEGF in lung cancer. Expert Opin Ther Targets 16: 395-406, 2012.

5. Teghom C, Giraud P, Menei P, et al: Renal carcinoma: point on treatment of brain metastasis. Bull Cancer 99: 627-634, 2012 (In French).

6. Wicki A and Rochlitz C: Targeted therapies in breast cancer. Swiss Med Wkly 142: w13550, 2012.

7. Dachs GU and Tozer GM: Hypoxia modulated gene expression: angiogenesis, metastasis and therapeutic exploitation. Eur J Cancer 36: 1649-1660, 2000.

8. Fang XC and Yu BY: Application of pyrolysis-high-resolution gas chromatography-pattern recognition to the identification of the Chinese traditional medicine mai dong. J Chromatogr 514: 287-292, 1990.

9. Yang PM AX: The initial research on Hupeh Liriope Root Tuber inducing HL60 cell differentiation. Dalian Med College J 14 37-41, 1992.

10. Mimaki Y, Takaashi Y, Kuroda M, Sashida Y and Nikaido T: Steroidal saponins from Nolina recurvata stems and their inhibitory activity on cyclic AMP phosphodiesterase. Phytochemistry 42: $1609-1615,1996$

11. Jeong A, Lee HJ, Jeong SJ, Lee EO, Bae H and Kim SH: Compound $\mathrm{K}$ inhibits basic fibroblast growth factor-induced angiogenesis via regulation of p38 mitogen activated protein kinase and AKT in human umbilical vein endothelial cells. Biol Pharm Bull 33: 945-950, 2010.

12. Tong Y, Zhang X, Tian F, et al: Philinopside A, a novel marine-derived compound possessing dual anti-angiogenic and anti-tumor effects. Int J Cancer 114: 843-853, 2005.

13. Sun L, Lin S, Zhao R, Yu B, Yuan S and Zhang L: The saponin monomer of dwarf lilyturf tuber, DT-13, reduces human breast cancer cell adhesion and migration during hypoxia via regulation of tissue factor. Biol Pharm Bull 33: 1192-1198, 2010.

14. Zhang Y, Liu J, Kou J, Yu J and Yu B: DT-13 suppresses MDA-MB-435 cell adhesion and invasion by inhibiting MMP-2/9 via the p38 MAPK pathway. Mol Med Rep 6: 1121-1125, 2012.

15. Jaffe EA NR, Becker CG and Minick CR: Culture of human endothelial cells derived from umbilical veins. Identification by morphologic and immunologic criteria. J Clin Invest 52: 2745-2756, 1973.

16. Lin S, Sun L, Hu J, et al: Chemokine C-X-C motif receptor 6 contributes to cell migration during hypoxia. Cancer Lett 279: 108-117, 2009

17. Lee OH, Kim YM, Lee YM, et al: Sphingosine 1-phosphate induces angiogenesis: its angiogenic action and signaling mechanism in human umbilical vein endothelial cells. Biochem Biophys Res Commun 264: 743-750, 1999.

18. Ashton AW, Yokota R, John G, et al: Inhibition of endothelial cell migration, intercellular communication, and vascular tube formation by thromboxane A(2). J Biol Chem 274: 35562-35570, 1999.
19. Chen L, Endler A and Shibasaki F: Hypoxia and angiogenesis: regulation of hypoxia-inducible factors via novel binding factors. Exp Mol Med 41: 849-857, 2009.

20. Wahl O, Oswald M, Tretzel L, Herres E, Arend J and Efferth T: Inhibition of tumor angiogenesis by antibodies, synthetic smal molecules and natural products. Curr Med Chem 18: 3136-3155, 2011.

21. Ma $\mathbf{J}$ and Waxman DJ: Combination of antiangiogenesis with chemotherapy for more effective cancer treatment. Mol Cancer Ther 7: 3670-3684, 2008.

22. Higa GM and Abraham J: Biological mechanisms of bevacizumab-associated adverse events. Expert Rev Anticancer Ther 9: 999-1007, 2009.

23. Chen HX and Cleck JN: Adverse effects of anticancer agents that target the VEGF pathway. Nat Rev Clin Oncol 6: 465-477, 2009.

24. Chakrabarti S, Rizvi M, Pathak D, Kirber MT and Freedman JE: Hypoxia influences CD40-CD40L mediated inflammation in endothelial and monocytic cells. Immunol Lett 122: 170-184, 2009.

25. Sluimer JC and Daemen MJ: Novel concepts in atherogenesis: angiogenesis and hypoxia in atherosclerosis. J Pathol 218: 7-29, 2009.

26. Izzard AS, Emerson M, Prehar S, et al: The cardiovascular phenotype of a mouse model of acromegaly. Growth Horm IGF Res 19: 413-419, 2009.

27. Verlohren S, Stepan H and Dechend R: Angiogenic growth factors in the diagnosis and prediction of pre-eclampsia. Clin Sci 122: 43-52, 2012

28. Nagamatsu T, Fujii T, Kusumi M, et al: Cytotrophoblasts up-regulate soluble fms-like tyrosine kinase-1 expression under reduced oxygen: an implication for the placental vascular development and the pathophysiology of preeclampsia. Endocrinology 145: 4838-4845, 2004

29. Forsythe JA, Jiang BH, Iyer NV, et al: Activation of vascular endothelial growth factor gene transcription by hypoxiainducible factor 1. Mol Cell Biol 16: 4604-4613, 1996.

30. Takahashi S: Vascular endothelial growth factor (VEGF), VEGF receptors and their inhibitors for antiangiogenic tumor therapy. Biol Pharm Bull 34: 1785-1788, 2011.

31. Shalaby F, Rossant J, Yamaguchi TP, et al: Failure of bloodisland formation and vasculogenesis in Flk-1-deficient mice. Nature 376: 62-66, 1995.

32. Zachary I and Gliki G: Signaling transduction mechanisms mediating biological actions of the vascular endothelial growth factor family. Cardiovasc Res 49: 568-581, 2001.

33. Gerber HP, McMurtrey A, Kowalski J, et al: Vascular endothelial growth factor regulates endothelial cell survival through the phosphatidylinositol 3'-kinase/Akt signal transduction pathway. Requirement for Flk-1/KDR activation. J Biol Chem 273 : 30336-30343, 1998 . 